\title{
Protons Induced Radiation Damage in Fast Crystal Scintillators
}

\author{
Fan Yang, Liyuan Zhang and Ren-Yuan Zhu \\ California Institute of Technology, Pasadena, CA 91125, USA \\ Jon Kapustinsky, Ron Nelson and Zhehui Wang \\ Los Alamos National Laboratory, Los Alamos, NM 87545, USA
}

\begin{abstract}
This paper reports proton induced radiation damage in fast crystal scintillators. A $20 \mathrm{~cm}$ long LYSO crystal, a $15 \mathrm{~cm}$ long $\mathrm{CeF}_{3}$ crystal and four liquid scintillator based sealed quartz capillaries were irradiated by $800 \mathrm{MeV}$ protons at Los Alamos up to $3.3 \times 10^{14} \mathrm{p} / \mathrm{cm}^{2}$. Four $1.5 \mathrm{~mm}$ thick LYSO plates were irradiated by $24 \mathrm{GeV}$ protons at CERN up to $6.9 \times 10^{15} \mathrm{p} / \mathrm{cm}^{2}$. The results show an excellent radiation hardness of LYSO crystals against charged hadrons.
\end{abstract}

Keywords: Crystal scintillators, LYSO, Proton damage

\section{Introduction}

Because of their superb energy resolution and detection efficiency, crystal scintillators are widely used in HEP experiments. A lead tungstate $\left(\mathrm{PbWO}_{4}\right.$ or $\left.\mathrm{PWO}\right)$ crys5 tal calorimeter, for example, has played an important role for the discovery of the Higgs boson by the CMS experiment [1]. One crucial issue is crystal's radiation damage in severe radiation environment. Cerium doped lutetium yttrium oxyorthosilicate $\left(\mathrm{Lu}_{2(1-x)} \mathrm{Y}_{2 x} \mathrm{SiO}_{5}\right.$ : Ce or LYSO) 10 crystals were chosen by the SuperB, Mu2e and COMET experiment to construct total absorption crystal calorimeters. They were also proposed as the active medium for a LYSO/W Shashlik calorimeter which was one of the two options proposed for the CMS forward calorimeter up- ${ }^{35}$ 15 grade $[2,3]$ for the HL-LHC. In this paper, we report an investigation on proton induced radiation damage in fast crystal scintillators.

\section{Samples and Experiment}

Table 1 lists six samples loaded on a remote controlled linear stage with a travel distance of $1 \mathrm{~m}$ at the Weapons Neutron Research facility of Los Alamos National Lab Neutron Research Center. They are a LYSO/W Shashlik cell, a box containing four liquid scintillator based quartz capillaries and four large size crystals: LFS, LYSO, BGO 25 and $\mathrm{CeF}_{3}$. The $800 \mathrm{MeV}$ proton beam has a Gaussian shape with a FWHM of about $2.5 \mathrm{~cm}$ at Los Alamos. Because of the $20 \mathrm{~cm}$ spacing between the samples multiple scattering effect to neighboring crystal is negligible.

Fig. 1 shows an optical fiber and lock-in amplifier based spectrophotometer used to measure longitudinal transmittance in situ for long crystal samples during and after the
Table 1: Samples loaded on the linear stage and the proton fluence

\begin{tabular}{c|c|c}
\hline Sample & Dimension $\left(\mathrm{cm}^{3}\right)$ & Fluence $\left(\mathrm{p} / \mathrm{cm}^{2}\right)$ \\
\hline \hline Shashlik Cell & $1.4 \times 1.4 \times 15$ & - \\
\hline Capillaries & $\phi 0.1 \times 6$ & $2.7 \times 10^{14}$ \\
\hline OET-LFS & $2.5 \times 2.5 \times 18$ & - \\
\hline SG-LYSO & $2.5 \times 2.5 \times 20$ & $3.3 \times 10^{14}$ \\
\hline SIC-BGO & $2.5 \times 2.5 \times 20$ & - \\
\hline SIC-CeF $_{3}$ & $2.2^{2} \times 2.6^{2} \times 15$ & $1.4 \times 10^{14}$ \\
\hline
\end{tabular}

irradiation. Because of a power blackout, only four quartz capillaries, the $20 \mathrm{~cm}$ LYSO crystal and the $15 \mathrm{~cm} \mathrm{CeF}_{3}$ crystal grown 20 years ago were irradiated to $2.7,3.3$ and $1.4 \times 10^{14} \mathrm{p} / \mathrm{cm}^{2}$ respectively, as shown in Table 1 . To avoid multiple Coulomb scattering and shower leakage in $20 \mathrm{~cm}$ long crystals, four LYSO plates of $14 \times 14 \times 1.5$ $\mathrm{mm}^{3}$ were irradiated by $24 \mathrm{GeV}$ protons at CERN from $7.4 \times 10^{13}$ to $6.9 \times 10^{15} \mathrm{p} / \mathrm{cm}^{2}$. All samples were measured 40 at Caltech before and about 80 days after the irradiation.

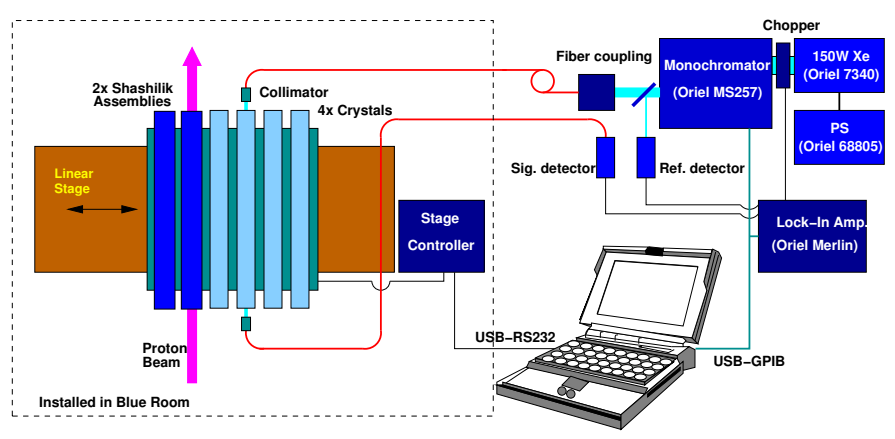

Figure 1: A schematic showing the experimental setup used to measure crystal's longitudinal transmittance in situ. 


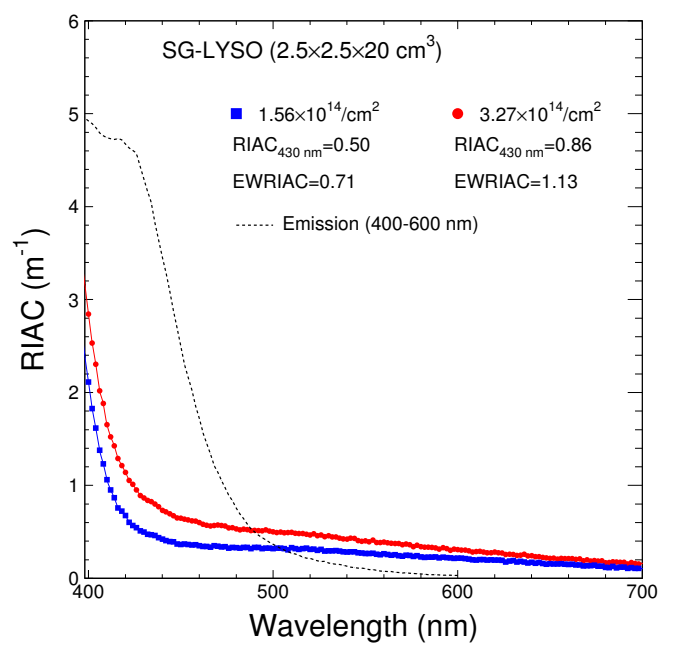

Figure 2: The RIAC spectra measured after each irradiation steps are shown for SG-LYSO.

\section{Results and Discussions}

No visible damage was observed in quartz capillaries after $2.7 \times 10^{14} \mathrm{p} / \mathrm{cm}^{2}$. The LYSO sample (SG-LYSO) was irradiated in two steps. Fig. 2 shows the radiation induced absorption coefficient (RIAC) spectra measured after 1.6 and $3.3 \times 10^{14} \mathrm{p} / \mathrm{cm}^{2}$. The emission weighted radiation induced absorption coefficient (EWRIAC) is about $1 \mathrm{~m}^{-1}$ after $3.3 \times 10^{14} \mathrm{p} / \mathrm{cm}^{2}$. Fig. 3 shows the RIAC values at $430 \mathrm{~nm}$ as a function of the proton fluence for this $20 \mathrm{~cm}$ long LYSO crystal and four $14 \times 14 \times 1.5 \mathrm{~mm}^{3} \mathrm{LYSO}$ plates irradiated by $24 \mathrm{GeV}$ protons at CERN from $7.4 \times 10^{13}$ to ${ }^{65}$ $6.9 \times 10^{15} \mathrm{p} / \mathrm{cm}^{2}$. Both data groups can be described well by linear fits. The factor of three difference between these two sets of data is caused by the multiple Coulomb scattering and hadronic shower leakage of $800 \mathrm{MeV}$ protons in the first nuclear interaction length of $20 \mathrm{~cm}$ in LYSO.

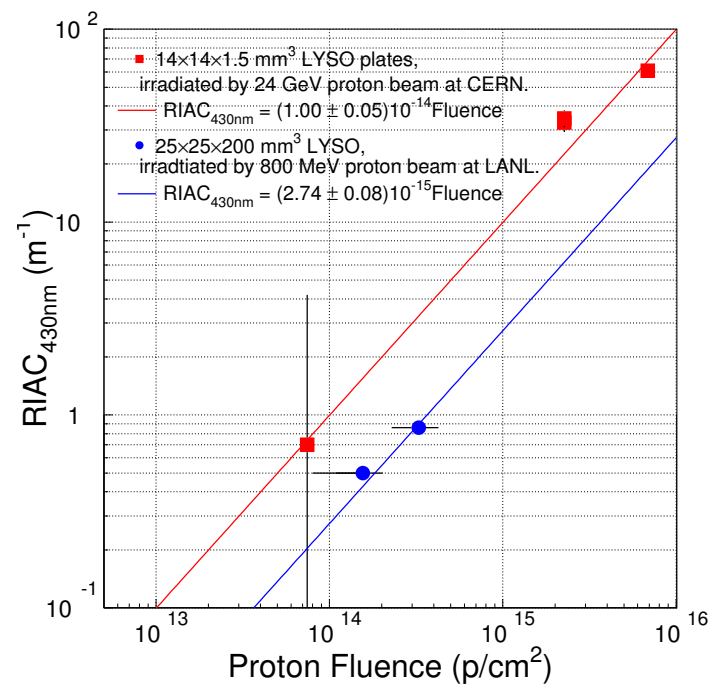

Figure 3: The RIAC at $430 \mathrm{~nm}$ are shown as a function of the proton ${ }^{85}$ fluence for LYSO bar/plates after $800 \mathrm{MeV} / 24 \mathrm{GeV}$ irradiation.

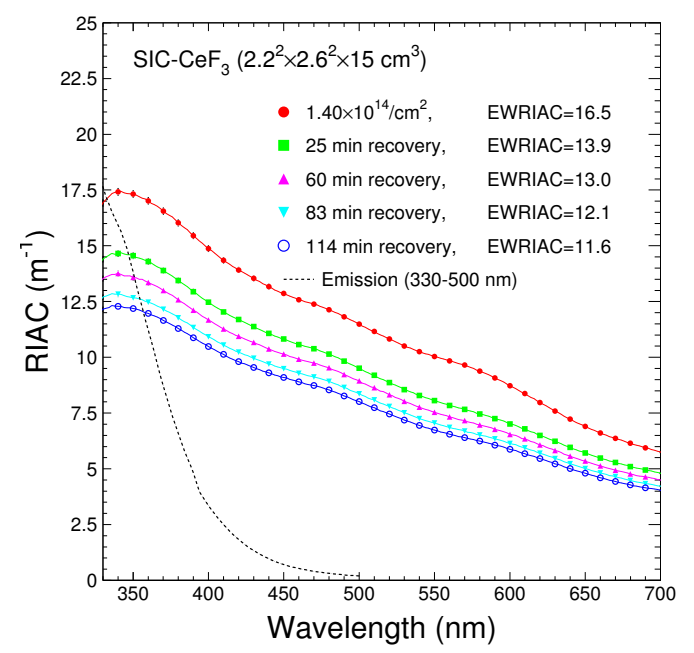

Figure 4: The RIAC spectra measured after irradiation are shown for $\mathrm{SIC}-\mathrm{CeF}_{3}$.

The $15 \mathrm{~cm}$ long $\mathrm{CeF}_{3}$ sample $\left(\mathrm{SIC}-\mathrm{CeF}_{3}\right)$ was irradiated to $1.4 \times 10^{14} \mathrm{p} / \mathrm{cm}^{2}$. Fig. 4 shows the RIAC spectra measured after irradiation and during recovery of about 2 hours. EWRIAC values of larger than $10 \mathrm{~m}^{-1}$ and a significant recovery from 16.5 to $1.8 \mathrm{~m}^{-1}$ were observed. Quality improvement is needed for this material to be used at the HL-LHC.

\section{Summary}

Three samples, a box containing four $6 \mathrm{~cm}$ long sealed quarts capillaries, a $20 \mathrm{~cm}$ long LYSO crystal sample and a $15 \mathrm{~cm}$ long $\mathrm{CeF}_{3}$ crystal sample, were irradiated by $800 \mathrm{MeV}$ protons at Los Alamos up to $3.3 \times 10^{14} \mathrm{p} / \mathrm{cm}^{2}$ with crystal's longitudinal transmittance measured in situ. Four $1.5 \mathrm{~mm}$ thick LYSO plates were irradiated by $24 \mathrm{GeV}$ protons at CERN up to $6.9 \times 10^{15} \mathrm{p} / \mathrm{cm}^{2}$. No visible damage was observed in quartz capillaries. The emission weighted radiation induced absorption in the $20 \mathrm{~cm}$ long LYSO crystal is about $1 \mathrm{~m}^{-1}$ after $3.3 \times 10^{14} \mathrm{p} / \mathrm{cm}^{2}$, which 75 is an order of magnitude smaller than that in the $15 \mathrm{~cm}$ long $\mathrm{CeF}_{3}$ sample. The result of these experiments provide important information for understanding proton induced radiation damage in fast crystal scintillators and their use in future HEP experiments at the energy and intensity frontiers.

\section{Acknoledgements}

This work was supported in part by the US Department of Energy Grant DE-SC0011925.

\section{References}

[1] CMS Collaboration, "Observation of a new boson at a mass of $125 \mathrm{GeV}$ with the CMS experiment at the LHC", Physics Letters B 716 (2012) 30 - 61. 
[2] R.-Y. Zhu, "The next generation of crystal detectors", Journal of Physics: Conference Series 587, 16th International Conference $90 \quad$ on Calorimetry in High Energy Physics (CALOR 2014), (2015) 012055. doi:10.1088/1742-6596/587/1/012055.

[3] L. Zhang, R. Mao, F. Yang and R.-Y. Zhu, "LSO/LYSO crystals for calorimeters in future hep experiments", IEEE Trans. Nucl. Sci. 61 (2014) 483 - 488. 